\title{
Formation of giant planets in disks with different metallicities
}

\author{
K. Kornet ${ }^{1, \star}$, P. Bodenheimer ${ }^{2}$, M. Różyczka ${ }^{1}$, and T. F. Stepinski ${ }^{3}$ \\ ${ }^{1}$ Nicolaus Copernicus Astronomical Center, Bartycka 18, Warsaw, 00-716, Poland \\ e-mail: [kornet; mnr]@camk.edu.pl \\ ${ }^{2}$ UCO/Lick Observatory, Department of Astronomy and Astrophysics, University of California, Santa Cruz, CA 95064, USA \\ e-mail: peter@ucolick.org \\ 3 Lunar and Planetary Institute, 3600 Bay Area Blvd., Houston, TX 77058, USA \\ e-mail: tom@lpi.usra.edu
}

Received 20 July 2004 / Accepted 20 September 2004

\begin{abstract}
We present the first results from simulations of processes leading to planet formation in protoplanetary disks with different metallicities. For a given metallicity, we construct a two-dimensional grid of disk models with different initial masses and radii $\left(M_{0}, R_{0}\right)$. For each disk, we follow the evolution of gas and solids from an early evolutionary stage, when all solids are in the form of small dust grains, to the stage when most solids have condensed into planetesimals. Then, based on the core accretion - gas capture scenario, we estimate the planet-bearing capability of the environment defined by the final planetesimal swarm and the still evolving gaseous component of the disk. We define the probability of planet-formation, $P_{\mathrm{p}}$, as the normalized fractional area in the $\left(M_{0}, \log R_{0}\right)$ plane populated by disks that have formed planets inside $5 \mathrm{AU}$. With such a definition, and under the assumption that the population of planets discovered at $R<5 \mathrm{AU}$ is not significantly contaminated by planets that have migrated from $R>5 \mathrm{AU}$, our results agree fairly well with the observed dependence between the probability that a star harbors a planet and the star's metal content. The agreement holds for the disk viscosity parameter $\alpha$ ranging from $10^{-3}$ to $10^{-2}$, and it becomes much poorer when the redistribution of solids relative to the gas is not allowed for during the evolution of model disks.
\end{abstract}

Key words. accretion, accretion disks - stars: planetary systems: protoplanetary disks - stars: planetary systems: formation

\section{Introduction}

Well over a hundred extrasolar planets have already been catalogued. It is very likely that radial velocity surveys performed in the solar neighbourhood have discovered a significant fraction of all Jupiter-like planets with periods shorter than $\sim 10 \mathrm{yr}$ (or, equivalently, with orbital radii smaller than $\sim 5 \mathrm{AU}$ ), whose parent stars exhibit periodic radial velocity variations with amplitudes $\geq 10 \mathrm{~m} \mathrm{~s}^{-1}$ Such a collection of planets provides a good data set with which predictions of different theories of planet formation can be compared (e.g. Ida \& Lin 2004).

Current observations strongly suggest that planet-bearing stars tend to have higher metallicities than field stars (Fig. 1). This correlation was reported for the first time by Santos et al. (2000), and later confirmed by Fischer \& Valenti (2003). The effect finds a natural explanation in the core accretion - gas capture (CAGC) model for giant planet formation. The model predicts a slow accretion of planetesimals onto a protoplanetary core until the mass of the core reaches a few $M_{\oplus}$. Then, gas accretion becomes significant, and around the core an extended envelope is formed, whose mass increases faster than that of the core. Eventually, the envelope becomes more massive than

\footnotetext{
^ Currently at MPIA, Königstuhl 17, 69117 Heidelberg, Germany.
}

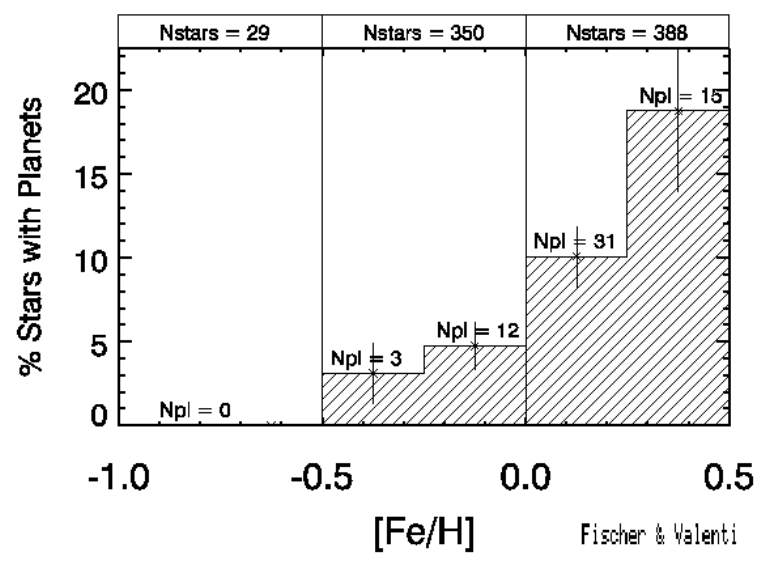

Fig. 1. The probability of planet occurrence as a function of the star's iron abundance (on the standard logarithmic scale with zero representing the solar value). Reproduced with permission from Fischer \& Valenti (2003).

the core, and a runaway accretion of gas ensues, which terminates when tidal effects become important or the protoplanetary disk is dissipated. The length of the core accretion phase decreases with increasing surface density of the planetesimal 
swarm (Pollack et al. 1996), which, in turn, is an increasing function of the original metal content of the protoplanetary disk and its central star. Consequently, the giant planets are expected to form more easily in disks with higher metallicities.

However, dust evolves due to different physical processes than the gaseous component of the disk (Weidenschilling \& Cuzzi 1993) As a result, a significant redistribution of solids takes place with respect to the gas, and, locally, the surface density of solids can be considerably enhanced compared to the initial one (Weidenschilling 2003; Stepinski \& Valageas 1997). Thus, statistical tests of the CAGC scenario must be based on evolving models of protoplanetary disks rather than static configurations which only serve as mass supply to planetary cores and envelopes. Such models must include the global evolution of solids. An extensive survey of such models was reported by Kornet et al. (2001), and applied to the problem of extrasolar planets formation by Kornet et al. (2002). In the latter paper it was found that effects of dust evolution allow for in situ formation of giant planets as close as 2 AU from the star. Here we obtain a much larger sample of disk models with different primordial metallicities, estimate how many of them could form planets, and compare the estimates to the observed correlation between stellar iron abundance and the probability of planet occurrence.

In Sect. 2 we briefly explain our approach to the evolution of a protoplanetary disk and planet formation. The results are presented in Sect. 3, while in Sect. 4 we summarize them and critically assess the simplifications on which our method is based.

\section{Method of calculation}

\subsection{The disk}

We model the protoplanetary disk as a two component fluid, consisting of gas and solids. The gaseous component is described by the analytical model of Stepinski (1998) which gives the surface density of gas, $\Sigma_{\mathrm{g}}$, as a function of radius $r$ and time $t$, in terms of a solution to the viscous diffusion equation. The viscosity coefficient is given by the standard $\alpha$ prescription. All other quantities characterizing the gas are calculated from a thin disk approximation, assuming vertical thermal balance. To take into account the effect of different metallicities on the structure of the gaseous disk, we scaled the opacity in Stepinski's model by a constant factor $Z$, independent of $r$ or $t$ and representing the metallicity of the disk expressed in solar units. Such a simple-minded approach is justified by the fact that the opacity in protoplanetary disks is mainly due to dust grains and molecules.

The main assumptions underlying our approach to the evolution of solids are (1) at each radius the particles have the same size (which in general varies over time); (2) there is only one component of dust, in this case corresponding to hightemperature silicates with the evaporation temperature $T_{\text {evap }}=$ $1350 \mathrm{~K}$ and bulk density $6.3 \mathrm{~g} \mathrm{~cm}^{-3}$ (this choice is justified by the fact that in most cases the surface density of a waterice planetesimal swarm would be too low in the range of distances considered here, i.e. $r \leq 5 \mathrm{AU}$, to enable formation of a giant planet in a reasonable time; see also Kornet et al. 2004); (3) all collisions between particles lead to coagulation; (4) when the temperature exceeds $T_{\text {evap }}$, local solids immediately sublimate and the vapor evolves at the same radial velocity as the gas component; (5) when the disk temperature falls below $T_{\text {evap }}$, local vapor immediately condenses into grains with a radius of $10^{-3} \mathrm{~cm}$ (minimum allowed by the numerical code); (6) the radial velocity of solid particles is entirely determined by the effects of gas drag; (7) the relative velocities of solid particles at which they collide are computed according to the turbulent model described by Stepinski \& Valageas (1997); (8) the evolution of solids does not affect the evolution of gas. At each radius, the vertical extent of the solid particle distribution is calculated and is evolved in time, so the effect of sedimentation of solids toward the midplane of the disk is taken into account. All assumptions and approximations are discussed in Stepinski \& Valageas (1997), Stepinski (1998) and Kornet et al. (2001).

The evolution of solids is governed by two equations. The first of them is the continuity equation for surface density of solid material, $\Sigma_{\mathrm{s}}$. The second one, describing the evolution of grain sizes, can be interpreted as the continuity equation for size-weighted surface density of solids, $\Sigma_{a} \equiv a(r) \Sigma_{\mathrm{s}}$, where $a(r)$ is the radius of solid particles at a distance $r$ from the star. The equations are solved numerically on a moving grid whose outer edge follows the outer edge of the dust disk. The details of the method can be found in Kornet et al. (2001).

We parameterize the initial conditions by the initial mass of the disk, $M_{0}$ (in $M_{\odot}$ ) and the decimal logarithm of the initial outer radius of the disk, $R_{0}$ (in $\mathrm{AU}$ ). From these two quantities the initial total angular momentum of the disk $j_{0}$, which we used in our previous calculations, can be calculated from the formula

$j_{0}=6.9 M_{0} R_{0}^{1 / 2} M_{\star}^{1 / 2}$

in units of $10^{52} \mathrm{~g} \mathrm{~cm}^{2} \mathrm{~s}^{-1}$, where $M_{\star}$ is the mass of the central star expressed in $M_{\odot}$ (see also Eq. (21) in Stepinski (1998). In the present survey $M_{\star}=1$. Once $M_{0}$ and $R_{0}$ are specified, the initial distribution of gas surface density $\Sigma_{\mathrm{g}, 0} \equiv \Sigma_{\mathrm{g}}(r, t=0)$, as well as the complete evolution of the gaseous disk, are obtained from the analytical model of Stepinski (1998).

We assume that at $t=0$ all solids are in the form of grains with radii $a=10^{-3} \mathrm{~cm}$, well mixed with the gas. Thus, the ratio of $\Sigma_{\mathrm{s}, 0}$ to $\Sigma_{\mathrm{g}, 0}$ is independent of $r$, and proportional to disk metallicity, $Z$. For a given metallicity, the initial surface density distribution of solids is

$\Sigma_{\mathrm{s}, 0}(r)=6 \times 10^{-3} Z \Sigma_{\mathrm{g}, 0}$,

where $Z$ is expressed in solar units.

\subsection{The planets}

We model the formation of a giant planet in situ, i.e. we do not include effects of migration, and the orbital parameters of the planet do not vary in time. Its evolution is followed according to the approach developed by Pollack et al. (1996) and Bodenheimer et al. (2000). The planet consists of a solid 
core and a gaseous envelope, both of which accrete mass from the disk. Our implementation of the evolutionary procedure is based on the following assumptions: (1) core accretion starts when all solids are in the form of planetesimals with the same radius $a=2 \mathrm{~km}$; (2) the surface density of solids in the feeding zone decreases with time as material accretes onto the core; (3) at each time the planetesimals are well mixed through the feeding zone of the planet; thus $\Sigma_{\mathrm{s}}$ is always uniform in space, but usually decreasing with time; (4) the planetesimals do not migrate into the feeding zone from outside, or vice versa, but they can be overtaken by the boundary of the feeding zone as it expands due to the growing mass of the planet.

With these assumptions, the minimum surface density of the planetesimal swarm $\Sigma_{\mathrm{s}, \mathrm{min}}$, needed to form a Jupiter-mass planet in less than $3 \mathrm{Myr}$ can be determined as a function of distance $r$ from the star. The details of the procedure are discussed in Kornet et al. (2002); here we only note that $2-\mathrm{km}$ planetesimals are on essentially Keplerian orbits, i.e. drag force has negligible effects on their distribution within the gaseous disk. The limit of $3 \mathrm{Myr}$ is supported by recent observations (e.g. Sicilia-Aguilar et al. 2004; Haisch et al. 2001), and it leaves a reasonable safety margin for the planet to complete its evolution before the canonical time of disk dispersal $\left(10^{7} \mathrm{yr}\right)$ elapses.

\section{Results}

To cover the range of masses and sizes of protoplanetary disks indicated by observations, we consider 11 values of $M_{0}$ uniformly distributed between 0.02 and 0.2 , and 10 values of $R_{0}$ so chosen that $\lg R_{0}$ is uniformly distributed between 1 and 4 . We evolve this set of models for seven different metallicities $(Z=0.2,0.5,0.75,1,1.5,2.0,2.5$ and 3.0$)$, and three values of the viscosity coefficient $\left(\alpha=10^{-3}, 10^{-2}\right.$ and $\left.10^{-1}\right)$. Using the procedure described in Sect. 2.1, we follow each model until all solids are in the form of "Keplerian" planetesimals or they have accreted onto the star. Next, each model with planetesimals is scanned to determine whether anywhere in the disk the surface density of the final planetesimal swarm, $\Sigma_{\mathrm{s}, \mathrm{f}}(r)$ exceeds $\Sigma_{\mathrm{s}, \min }(r)$. Models with a range of $r$ such that $\Sigma_{\mathrm{s}, \mathrm{f}}(r) \geq$ $\Sigma_{\mathrm{s}, \min }(r)$ are planet bearing; however for further analysis only those that form planets closer than $5 \mathrm{AU}$ from the star are selected. We introduce this limitation in order to account for the fact that (as we already mentioned in Sect. 1) the sample of observed extrasolar planets is reasonably complete to $\sim 5 \mathrm{AU}$ only.

For given $\alpha$ and $Z$ our grid of $M_{0}$ and $\lg R_{0}$ may be thought of as a nonrandom sample drawn from the unknown ensemble of disk masses and radii. Let $\mathcal{R}_{p, 5}$ be the region in the $\left[M_{0}, \lg R_{0}\right]$ plane occupied by models that form planets at $r \leq 5$ AU from the star, and let $A_{p, 5}$ be the area of $\mathcal{R}_{p, 5}$. The rate of planet occurrence within the sample is given by the ratio

$P_{\mathrm{p}}=\frac{A_{p, 5}}{A}$,

where $A$ is the area of the region occupied by the whole grid of models.

The results are presented in Fig. 2. It shows the rate of planet occurrence as a function of disk metallicity for different

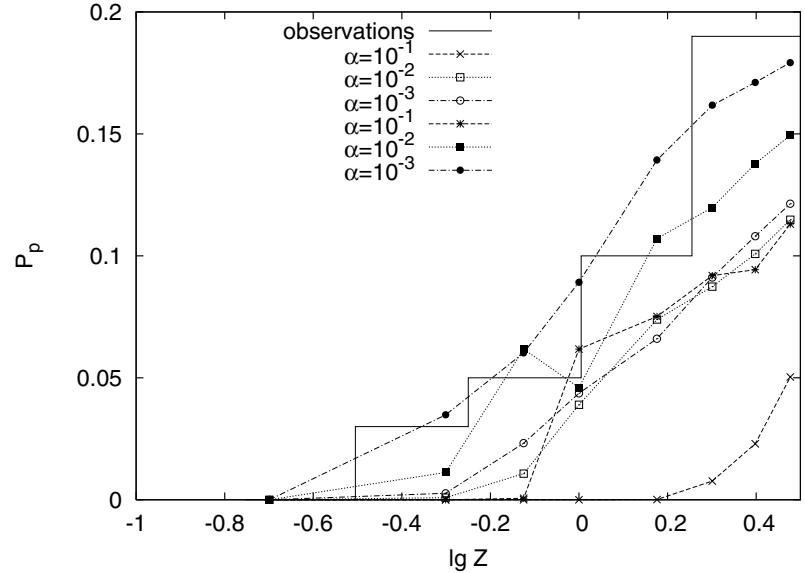

Fig. 2. The rate of planet occurrence as a function of the primordial metallicity of protoplanetary disks. Lines marked with different symbols are obtained for different values of $\alpha$, as labeled in the upper left corner. Filled symbols: models with the redistribution of solids; open symbols: models with a constant $\Sigma_{\mathrm{s}} / \Sigma_{\mathrm{g}}$ ratio. The histogram shows the observational data.

values of $\alpha$. As one might expect, $P_{\mathrm{p}}$ is an increasing function of $Z$ : in more metal-rich disks there is more solid material, so the planets form more easily. We believe that deviations from that rule result from the poor resolution of our grid of models.

There is also a monotonic trend seen when $P_{\mathrm{p}}$ is considered as a function of $\alpha$ at constant $Z$ : giant planets form more easily in less viscous disks. This is because for every $\alpha$ and every $Z$ the lower border of the planet-bearing region runs very close, and nearly parallel, to the line $\mathcal{L}$ that separates models in which all solids accrete onto the star from those in which some solids survive (see Fig. 3). The observed trend results from two competing factors. On the one hand, to a good approximation $\mathcal{L}$ marks the location of models in which the initial temperature at the outer edge of the disk is equal to $T_{\text {evap }}$ (Kornet et al. 2001, 2002). Among disks with the same $M_{0}$ and $R_{0}$, those with smaller $\alpha$ are cooler as a result of lower energy generation rate. Thus, when $\alpha$ decreases, $\mathcal{L}$ must move toward smaller values of $R_{0}$, and the area of the planet-bearing region increases. On the other hand, decreasing $\alpha$ reduces the maximum $R_{0}$ for which planet formation is possible in disks with the same $M_{0}$. This is because in cooler disks the solids gain smaller inward velocities (Weidenschilling 1977; Kornet et al. 2004). As a result, for the same disk parameters except for a lower $\alpha$, the redistribution of solids is less efficient, and the final planetesimal swarm is more extended. A more extended swarm implies lower surface density of solids and less favorable conditions for planet formation. In particular, when we move on the $\left[M_{0}, \lg R_{0}\right]$ plane along an $M_{0}=$ const line such that $R_{0}$ increases, we leave the planet-bearing region sooner (in other words, decreasing $\alpha$ causes the upper boundary of the planetbearing region to move downwards on the $\left[M_{0}, R_{0}\right]$ plane). Our calculations show that the first of these two effects is the dominant one. Therefore, for a given metallicity the fraction of disks with giant planets increases with decreasing $\alpha$.

To illustrate the importance of the redistribution of solids for the process of planet formation, we calculated $P_{\mathrm{p}}$ for the 


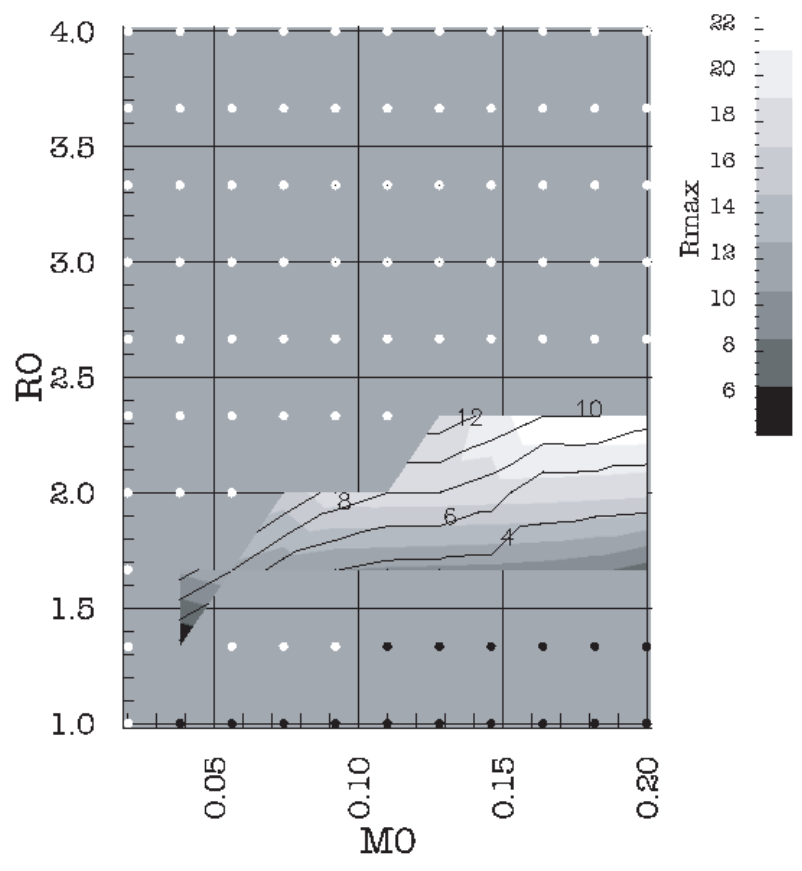

Fig. 3. As functions of the initial disk mass $M_{0}$ (in solar masses) and the logarithm of the initial radius $R_{0}$ in astronomical units, the contours and grey scale, respectively, give the inner and outer radius of the region around the central star where giant planet formation is possible in a maximum of $3 \mathrm{Myr}$. White circles indicate disk models where the solid surface density is everywhere below the critical value for planet formation. Black circles indicate disks in which all of the solid material accretes onto the star. The disk viscosity parameter is $\alpha=1 \times 10^{-2}$, and the primordial metallicity is solar.

same grid of initial conditions, but based on $\Sigma_{\mathrm{s}, 0}$ instead of $\Sigma_{\mathrm{s}, \mathrm{f}}$. The results are shown in Fig. 2. As expected, the redistribution of solid material is most important for $\alpha=10^{-1}$, but even for $\alpha=10^{-3}$ it cannot be neglected. For example, with $\alpha=$ $10^{-3}$ and $Z=3$, including redistribution increases the ratio of planet occurrence by a factor $\approx 1.5$. For lower metallicities the effect is even stronger. In particular, it causes $Z_{\min }$, the minimum $Z$ at which planets can form, to decrease from $\sim 0.45$ to $\sim 0.25$ and from $\sim 1.4$ to $\sim 0.7$, respectively, for $\alpha=10^{-3}$ and $10^{-1}$. It can be seen that even in low-metallicity disks $\Sigma_{\mathrm{s}}$ can locally grow above $\Sigma_{\mathrm{s} \text {,min }}$, creating conditions favorable for planet formation.

With $\alpha=10^{-3}$ and the redistribution of solids included, the calculated rate of occurrence of planets is in good agreement with observational data. Obviously, it is a mere coincidence, without any practical meaning. Our rates strongly depend on the extent and position of the grid of initial conditions in the $\left[M_{0}, R_{0}\right]$ plane. Suppose that we increase the maximum $\lg R_{0}$ above the considered value of 4 . The rate of planet occurrence would decrease, because we would add only models in which $\Sigma_{\mathrm{s}, \mathrm{f}}$ is not high enough to satisfy the requirements for planet formation. To make our results independent of this effect, for every value of $\alpha$ we normalized $P_{p}(Z)$ in such a way as to reproduce the observational value of $\sim 0.2$ for $Z \approx 3$. The normalized rates are shown in Fig. 4. Now, curves for both $\alpha=10^{-3}$ and $10^{-2}$ fall in the range of observational values, while disks with $\alpha=10^{-1}$ still produce too few planets

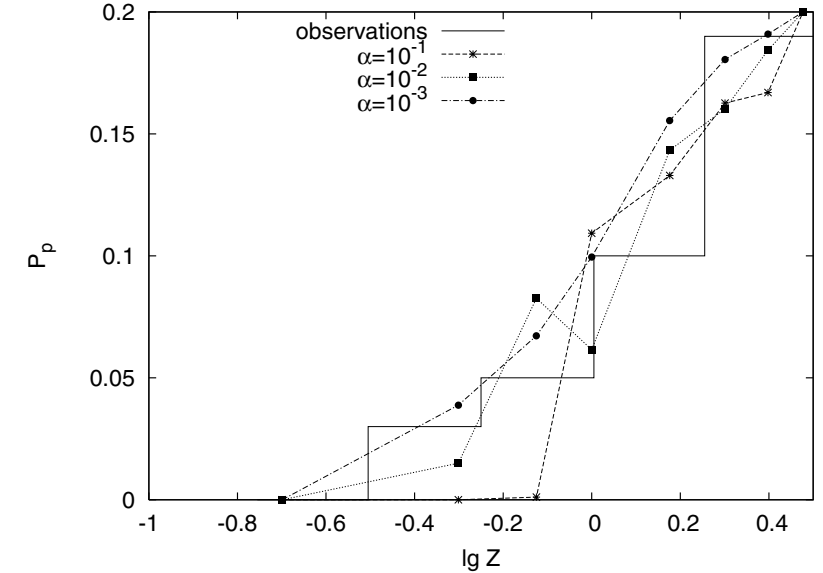

Fig. 4. Rate of planet occurrence as a function of the primordial metallicity in models with the redistribution of solids included. Same as in Fig. 2, but all curves are normalized to match the observational data for $\lg Z=0.48$.

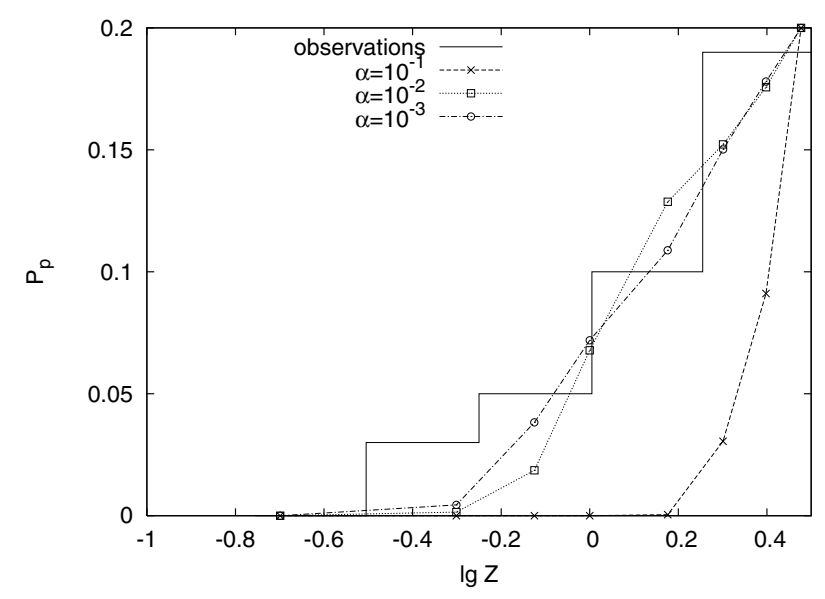

Fig. 5. Rate of planet occurrence as a function of the primordial metallicity in models without the redistribution of solids. Same as in Fig. 2, but all curves are normalized to match the observational data for $\lg Z=0.48$.

for small values of $Z$. For comparison, in Fig. 5 we shows the normalized rates obtained from models without the redistribution of solids. While for $\alpha=10^{-3}$ and $10^{-2}$ they are consistent with the observational data for metal rich disks, they are significantly lower for small values of $Z$, and they give too high values of $Z_{\min }$. For $\alpha=10^{-1}$, the results do not match observations except for $Z \sim 3$, where the agreement is enforced by the normalization procedure.

\section{Discussion and conclusions}

Based on a simple approach to the evolution of solids in protoplanetary disks we calculated rates of giant planet occurrence on orbits smaller than $5 \mathrm{AU}$. We find that in more metal-rich disks the planets form more easily, and we qualitatively reproduce the observed correlation between the probability that a giant planet would be found at a star and the metal content of that star. We are able to match this correlation quantitatively for disk models with the viscosity parameter $\alpha$ ranging from $\sim 10^{-3}$ 


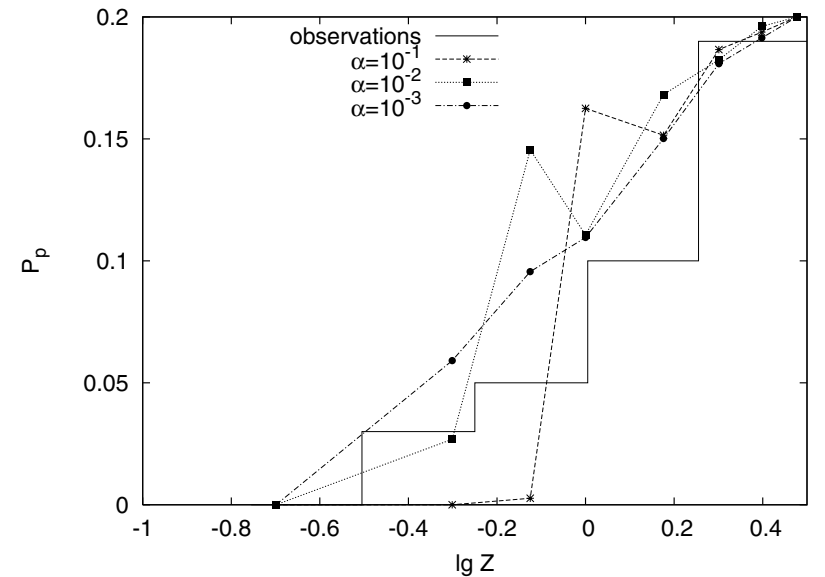

Fig. 6. Same as Fig. 4, but based on disk models with initial parameters uniformly distributed on the $\left[M_{0}, \lg j_{0}\right]$ plane.

to $\sim 10^{-2}$. We also show that in the low-metallicity regime the calculated occurrence rates agree with the observed ones only if the redistribution of solids is included in the models. In such models the surface density of solids locked in the final planetesimal swarm can be locally much higher than the initial surface density of the dust. This effect is particularly important if we want to match the observed value of limiting stellar metallicity $Z_{\min }$ below which no planets have been detected. Again, only models which allow for the redistribution of solids are successful, while models which do not include this effect produce $Z_{\min }$ which is significantly too high. This finding supports our approach to the evolution of protoplanetary disks, whose key ingredient is the radial drift of solids due to the gas drag.

The above conclusions are based on the assumption that our grid of models is a representative sample of the real distribution $\mathcal{P}\left(M_{0}, R_{0}\right)$ of initial masses and radii of protoplanetary disks, which is not known. Such an assumption is acceptable provided that the real disks are uniformly (or almost uniformly) distributed on the $\left[M_{0}, \lg R_{0}\right]$ plane. Adopting it we demand that very large disks be relatively rare, which seems to be an entirely reasonable requirement (note that $\mathcal{P}\left(M_{0}, R_{0}\right)$ must approach 0 for $R_{0} \rightarrow \infty$ ). We tested the sensitivity of our results to the way the initial parameters are sampled by repeating the calculations with initial models uniformly distributed on the $\left[M_{0}, \lg j_{0}\right]$ plane. The results, normalized to the observational data the same way as before, are shown in Fig. 6 . The agreement is poorer, but the same general trend is still well visible. Moreover, we see that $Z_{\min }$ does not depend on the sampling procedure (this conclusion is restricted to samples which do not include extremely massive disks with $M_{0}>0.2 M_{\odot}$; note however that such disks would most likely be gravitationally unstable).

Our results are based on a highly simplified scenario of the evolution of solids in protoplanetary disks. The assumption concerning the size distribution of solid particles, according to which at each distance from the star all particles have the same diameter, seems to be particularly radical. However, detailed work by Morfill (1985), Mizuno et al. (1988) and Weidenschilling (1997) showed that the distribution of solids quickly converges to a stage in which most of the mass is concentrated in a narrow range of sizes at the momentary maximum size. Their conclusion has recently been corroborated by the behaviour of solids in the two-dimensional disk model by Weidenschilling (2003). Thus, despite its simplicity, our our approach to the size disitribution function can be regarded as a reasonable approximation.

Our next radical assumption is the $100 \%$ efficiency of coagulation In reality, one hardly expects that collisions between solid particles always lead to sticking without fragmentation. However, calculations reported by Kornet et al. (2004) show that if the main process responsible for particle growth is collisional coagulation, then its efficiency must be high - otherwise the disk would rapidly lose most of its solid material, disabling planet formation or severely diminishing its possibility. Note also that recent observations by Chakraborty et al. (2004) indicate that asteroid-sized bodies can exist in disks as young as 0.1 Myr. Again, if they have grown due to coagulation, the sticking efficiency must have been very high.

Migration of planets due to gravitational interactions with the disk is not included in our approach. Obviously, we do not claim that the process of migration does not operate. On the contrary, we have to assume some form of redistribution of planets in order to explain the origin of "hot Jupiters", whose orbits are much tighter than the tightest orbits allowed by our models ( $\sim 1 \mathrm{AU})$. However, as long as we are interested only in global rates of planet occurrence, and not in the distribution of orbital parameters, our results remain valid provided that the number of planets which migrate to $r<5$ AU from larger orbits is negligible compared to the number of planets which are born there.

The fact that such a simple model can reproduce the available data has two possible interpretations. According to the pessimistic one we observe a mere coincidence which does not have any physical meaning. According to the optimistic one we identify the most important process governing the evolution of solids in a protoplanetary disk (radial drift, induced by the gas drag, and associated with collisional coagulation), and we provide support for the core accretion - envelope capture scenario of giant planet formation. More sophisticated disk models based on fewer assumptions, which we are presently working on, should help us decide which of the two is true.

Acknowledgements. KK and MR were supported by the grant No. 1 PO3D 02626 from the Polish Ministry of Science. They gratefully acknowledge benefits from the activities of the RTN network "PLANETS" supported by the European Commission under the agreement No HPRN-CT-2002-00308. This work was supported in part by the NASA Origins of Solar Systems program through grant NAG 5-13285 to the University of California, Santa Cruz. KK was also supported by the German Research Foundation (DFG) through the Emmy Noether grant WO 857/2-1. TS was supported by the LPI, which is operated by USRA under contract CAN-NCC5-679 with NASA. This is LPI contribution No. 1214.

\section{References}

Bodenheimer, P., Hubickyj, O., \& Lissauer, J. J. 2000, Icarus, 143, 2 Chakraborty, A., Ge, J., \& Mahadevan, S. 2004, ApJ, 606, L69 
Fischer, D., \& Valenti, J. A. 2003, in Scientific Frontiers in Research on Extrasolar Planets, ASP Conf. Ser., ed. D. Deming, \& S. Seager (San Francisco: ASP), 294, 117

Haisch, K. E., Lada, E. A., \& Lada, C. J. 2001, ApJ, 553, L153

Ida, S., \& Lin, D. N. C. 2004, ApJ, 604, 388

Kornet, K., Stepinski, T. F., \& Rozyczka, M. 2001, A\&A, 378, 180

Kornet, K., Bodenheimer, P., \& Rozyczka, M. 2002, A\&A, 396, 977

Kornet, K., Stepinski, T. F., \& Rozyczka, M. 2004, A\&A, 417, 151

Mizuno, H., Markiewicz, W. J., \& Voelk, H. J. 1988, A\&A, 195, 183

Morfill, G. E. 1985, in Birth and Infancy of Stars, ed. R. Lucas, A. Omont, \& R. Stora (Amsterdam: Elsevier Science Publishers), 693

Pollack, J. B., Hubickyj, O., Bodenheimer, P., et al. 1996, Icarus, 124, 62
Santos, N. C., Israelian, G., \& Mayor, M. 2000, A\&A, 363, 228

Sicilia-Aguilar, A., Hartmann, L. W., Briceño, C., Muzerolle, J., \& Calvet, N. 2004, AJ, 128, 805

Stepinski, T. F. 1998, Icarus, 132, 100

Stepinski, T. F., \& Valageas, P. 1997, A\&A, 319, 1007

Weidenschilling, S. J. 1977, MNRAS, 180, 57

Weidenschilling, S. J. 1997, Icarus, 127, 290

Weidenschilling, S. J. 2003, in Lunar and Planetary Science Conf., \#34, abstract \#1707

Weidenschilling, S. J., \& Cuzzi, J. N. 1993, in Protostars and Planets III, ed. E. Levy, \& J. Lunine (Tuscon: Univ. of Arizona Press), 1031 